\title{
Multicast Cooperative Routing For Opportunistic Data Transfer in Mobile Ad Hoc Network
}

\author{
K.Vanisrsee ${ }^{1}$, DR.V.S.K.Reddy ${ }^{2}$ \\ ${ }^{l}$ (ECE Prof.in Engineering College India) \\ ${ }_{2}^{2}$ (Principal and Professor of ECE Dept, Malla Reddy College of Engineering and Technology, India)
}

\begin{abstract}
In wireless networks, especially in mobile ad hoc networks nodes are typically battery powered, and also networks are noisy and interference rich environment, so it is often slow downed by weak communication link. Hence in this research paper consider both physical layer cooperative communications in order to design network layer routing algorithms that are energy efficient. Let us consider cooperative diversity from the physical layer to improve the results from reliability, Routing at community layer, and formulate minimal power routing as a joint optimization of the transmission power at the bodily layer and the link selection at the network layer. The Efficiency of the Wi-Fi hyperlink may be growth by means of Multicasting through sending single reproduction of messages to all group members. Multicast transmission is a more effective mechanism when compared to unicasting, in terms of increased bandwidth, balanced traffic load, balanced power consumption, reduced delay between the days and robustness of data delivery.Here Nodes inside the network use a lightweight proactive source routing protocol to determine a listing of intermediate nodes that the information packets ought to follow en route to the vacation spot.In traditional unicast transmission can be picked by multiple nodes, but only one of them is the intended receiver (destination node or relay). If this node has did not acquire the transmission, the packet needs to be resent again. In effect, all nodes which have acquired the transmission that are toward the vacation spot than the transmitter may be relays, so it isn't always necessary to rely upon a particular node to forward the data in principle. This method can save a great deal of resources which are compared to old methods and considered in network protocol design, known as multipath cooperative routing techniques for Opportunistic data transfer between source and destination nodes. This is extension of unicast Opportunistic Cooperative Routing in Mobile Ad hoc Network (O-CORMAN) scheme. The proposed work display that a higher performance in phrases of throughput, energy, controls overhead when compared with unicast o-corman and also than existing non cooperative scheme.
\end{abstract}

Keywords: cooperative communication, diversity, multicast routing, o-corman, Opportunistic data transfer

\section{Introduction}

Data Tranfer in Mobile Ad-hoc Networks (MANETs) now a days a highly used in maximizing the employment in both the commercial network situations and military, where infrastructure is costly and dangerous to deploy. Many MANETs applications need the nodes to work as a group to carry out a data transmission from various source to certain point this can be done by using multicasting scheme, hence multicasting plays a major part in MANETs. This kind of application is efficient because of broadcasting nature of cellular (wireless) network for it can enhance the efficiency of the wireless links.As anEnd result, multicast routing has turn out to be a studies cognizance these days, Multicast communication as defined, is an effective approach of distributing statistics to a set of individuals such as virtual classroom, remote conference and distributed games, it permits a single IP datagram to be routed to multiple hosts. MANETs consists of more number of mobile nodes and also source node is always away from destination node, hence it requires more transmission strength and bandwidth to preserve correct routing facts;in the network the results achieved from the communication between the routing and nodes information, by using this process it consumes more battery power. Many nodes in broadcast region of the wire-less communication introduce interference this reduces the signal to noise ratio to the fixed node, the resultant receiving performance is graded. With the help of cooperative communication, the transmitted power level at source node reduced by the introduction of cooperative relay. 


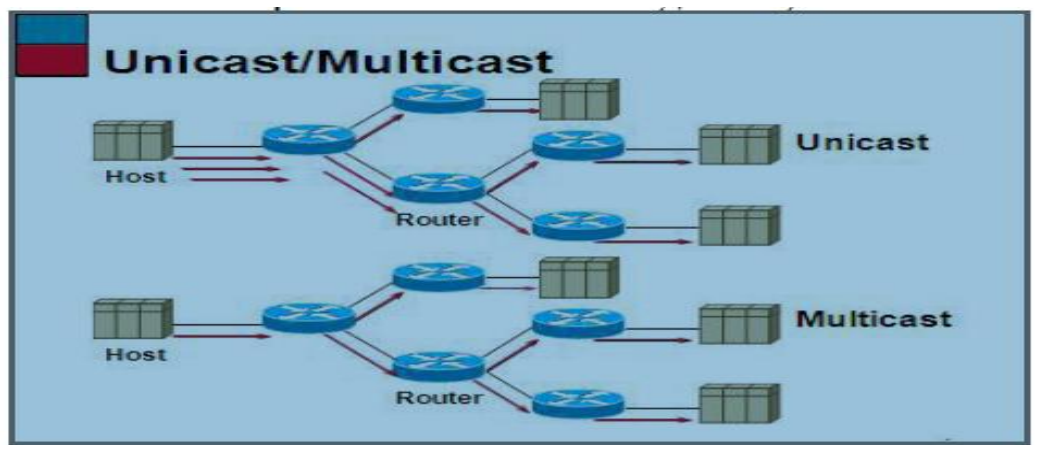

Fig1: path way of Multicast and Unicast

In a vigorous environment topology changes unpredictably, to control the topology [1] needed to determine appropriate topology which decreases the energy consumption, interference will be decreased and the lifetime will be increased MANET . The quality of topology is measured by using how the nodes are connected, energy efficient data forwarding, and throughput. The Linking protocols are designed over an appropriate topology [2]. In a network nodes are connected and act as routers, to maintain the constant data packets. Routes contain links which are connected between the two nodes. Due to mobility of nodes which can cause broken routing links and nodes to retransmit their information based on routing .This consumes more power and additional overhead traffic under the network routing information. The O-CORMAN occurs smaller than AODV by including forward lists in data packets. The route quality is influenced by change in link quality. A varying link route does not produce good results.

The network layer has received the most attention when working on MANETs. The two most important operations at the network layer, i.e., data forwarding and routing, are distinct concepts. Data forwarding regulates how packets are taken from one link and put on another. Routing determines the path in which a data packet should follow the data from source node to destination. The routing provides the data forwarding with control input, Due to multipath propagation, fading and interference affect reliability of the Adhoc network by introducing the cooperative diversity at the physical layer improves reliability in the case of outage probability. By contrast, the cooperative communications used to increase the transmission rate.

Routing protocols can be either unicast or multicast depends on the number of routes discovered [3].In a unicast data packets are send through single best path from the source node to the destination node,and unable to balance traffic [4]load. If the path fails a new route discovery must be initiated resulting in significant delay and packet loss,where as in multicast maintain all routes that can be detected during a route discovery process, They are better for load balancing.

\subsection{Multicasting}

Multicasting is group-oriented computing in which the transmission of data packets are more than one node, sharing one multicasting address. The senders and the receivers form the multicast group. When designing multicast routing protocols for ad hoc network, some of the key issues should be considered it includes constant update of the delivery paths, dynamic group membership, and as little state information as possible. Multicast routing protocols have been proposed for MANET, can be classified as according to the kind of routes they create tree-based or mesh based.

Multicast protocols select more than one path to reach the destination. They are better for load balancing than unicast protocols. Multicast routing improves the communication efficiency and also improves Quality of Service by using different paths simultaneously and also more reliable,reduce frequent routing update robust and consequently reduces the control overhead, it enhances the data transmission rate, network bandwidth is increased and the energy is saved [6].

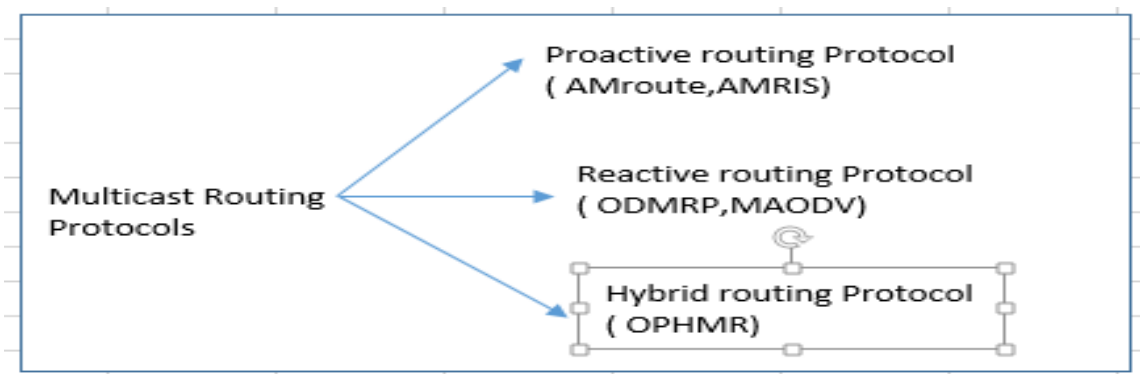

Fig2: Multicast Routing Protocols Classification 
In this paper tree based multicast routing protocol is used for data transmission, in this node maintain the information table for each node which are in the network. This protocol is called as table driven protocol. Contribution of o-corman scheme as follows

- In this multicast proactive routing protocol packets can be divided and sent simultaneously, depends on number path is created to reach destination.

- The intermediate node can adjust the routing information while data packets are deliver from source to destination. If an intermediate nodes have a knowledge about new routes, it is able to use this new route for date forwarding. It means that new which is forwarded is replaces the old list.Such updated information is propagated upstream quickly without any additional overhead. As a result, all upstream nodes learn about the new route at a rate much faster than via periodic route exchanges.

- Opportunistic data forwarding is taken to another level by allowing nodes that are not listed as intermediate forwarders to retransmit data if they believe certain packets are missing.

\section{Related Work}

The use of the broadcasting nature of wireless channels at the link layer, ExOR [7] is a cross- layer explorative opportunistic proactive routing protocol is used for data forwarding in multi-hop wireless networks by Biswas and Morris. A sender broadcasts a batchof packets each packet contains a list of forwarded nodes. In [8] proposes a new simple opportunistic adaptive routing protocol (SOAR) to overcome the draw backs in [9] author takes the transmission based on nodes instead of next hop method called priority-based forwarding. MORE [10] improves ExOR, to further increase the spatial channel reuse in a single flow via intra flow network coding to reach destination from the source. To genralize the idea behind ExOR to mobile wireless networks and other different types, a lightweight routing algorithms with proactive source routing capabilities are preferred. in [11] solve the problem of opportunistic data transfer in MANETs.The solution is called Cooperative Opportunistic Routing in Mobile Ad hoc Networks (CORMAN).It is an extension of EXOR, pure network layer scheme that can be built atop off-the-shelf wireless networking equipment. Nodes in the network use a lightweight proactive source routing protocol to determine a list of intermediate nodes that the data packets should follow en route to the destination.In [12], ODMRP (On Demand Multicast Routing Protocol) is proposed ,ituses the concept of "forwarding Node" to do the multicasting and it finds the forwarding node in the network. Only this node will forward multicast messages until it reaches a group member, then creates a joint table message and broadcast to its neighbors. This neighbor node establish forwarding node. In [13] MAODV (Multicast On-demand Distance Vector routing protocol) broadcast to find, the route in on demand basics and constucts a routing tree. The AODVM presents [14] loop free and node disjoint paths. The CM AODV [15] selects nodes based on signal to noise interference ratio. To improve the throughput and delay, the Multipath Source Routing [16] use the weighted round robin packet distribution technique. The split Multipath Routing [17] selects the disjoint multiple routes based on limited hop counts. Node Disjoint Multipath Routing (NDMR) gives multi path [18] with disjoint nodes.

\section{Proposed Technique}

The proposed routing scheme is the extension of O-CORMAN unicast. In this proposed work data transfer are done with using many simultaneous flows, and the network resources are well shared. The coordination among, multiple qualified small scale retransmitters can be achieved with better link stability. The three modules are Multipath Proactive Source Routing, Large Scale Live Update and Small Scale Retransmission. Figure3shows Over view of o-corman and figure 4 tells about the Live Update and Retransmission .

\subsection{Multipath Proactive Source Routing}

According to the proactive source routing the nodes periodically exchange the topology structure information with each other. It converges when the number of iterations is equal to the network diameter. Thus each node has the path structure information indicating the path to all other nodes. When a source wants to communicate with the destination first it finds multiple paths to the receiver. It forms a matrix based on the possible paths available, and finds number of disjoint paths available [3]. After finding the available disjoint paths the packets are then transferred towards the destination. Every node has an iteration of $\mathrm{O}(\mathrm{n})$ when it broad cast the information this overhead is same in distance vector algorithms and much smaller than that in link state algorithms. 


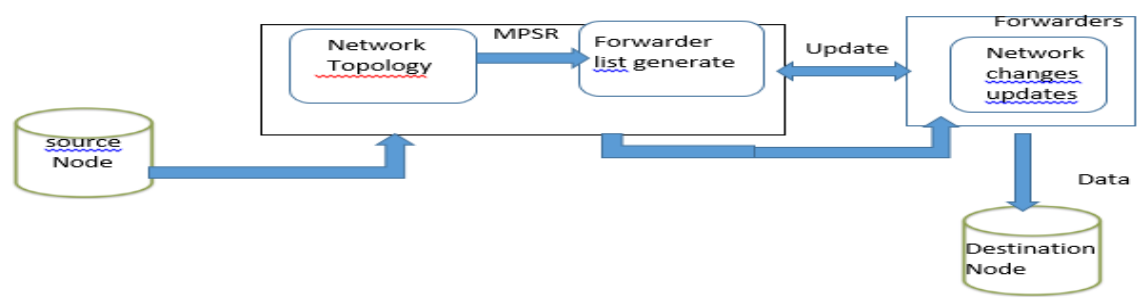

\subsection{Large Scale Live Update}

Fig3: Over view of o-corman

As per the already per defined routing, each packet through which is transferred. But when the data packets are received by a forwarding node, it may have a different view of how to forward the data to the receiver. The intermediate nodes aware of new route and data is forwarded through new route and old route is replaced by new route. Since the forwarding node in the forwarder list is, close to the destination than the source node then the forwarding node has more updated routing information. In this case from this point on the forwarding node can update the forwarder list in the packets, according to its own knowledge ie when the network diameter is large and when nodes are moving fast, the routing information can be outdated by the time it reaches a remote node. When the destination node is far away from forwarder list node, this nodes have less knowledge about the network structure. Thus the forwarder list sent by the source needs to be adjusted as the packets are forwarded towards the destination. A node that is on the forwarder list sent by the source and which has the higher priority and has received any packet is called the frontier. Only the frontier updates the new list which has been sent forward and also only the segment of the list between the frontier and the destination is updated. A node which is no longer a forward node should have only incorporate forward list that it hears from the downstream nodes which is used to avoid unwanted updates. The nodes which are in the upstream can overhear these packets and the new list which has been sent forward and they update their own routing information. This information is incorporated when forwarding their fragments. This backtrack will continues until the source node is known about latest route information

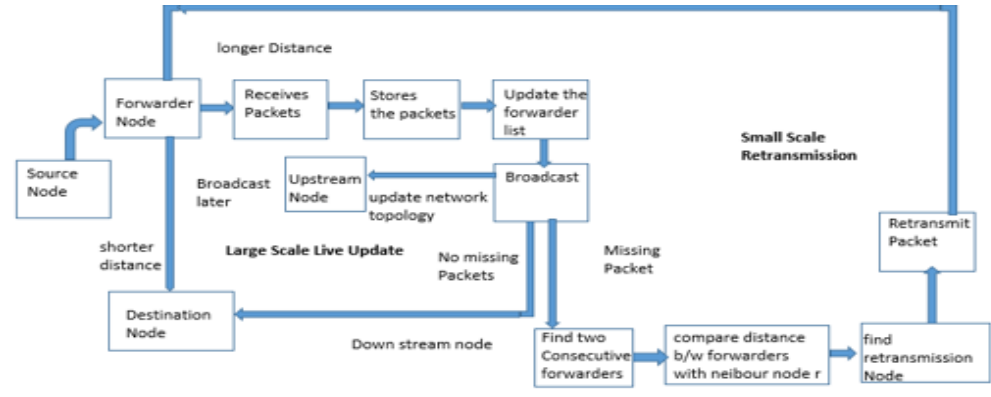

\subsection{Retransmission}

Fig 4: Route update and small scale retransmission

It is a technique to enhance the reliability of packet transmission between two consecutive listed forwarders. Nodes which are not in the sent forwarder list but positioned between this two consecutive forwarders that can retransmit data when the data are not received successfully. Consider figure 2 , the two consecutive forwarders on this list such as $\mathrm{f} 1$ and $\mathrm{f} 2$. Let $\mathrm{f}$ be a node present somewhere in between $\mathrm{f} 1$ and $\mathrm{f} 2$. After $\mathrm{f} 2$ has transfered its fragment of packets and by comparing the packets transfered by $\mathrm{f} 1$ to $\mathrm{f} 2$, node $\mathrm{r}$ knows that the packets $\mathrm{f} 2$ has missed. It is now qualified to retransmit these packets that are believed to be missing. If there are more than one such $\mathrm{f}$ in that area, such as $\mathrm{r} 1$ and $\mathrm{r} 2$, then at most one such node should retransmit. For a node $f$ most one node should retransmit in the case it should follow the following conditions. 1. The node $\mathrm{f}$ should be a neighbour of both $\mathrm{f} 1$ and $\mathrm{f} 2$. 2 . The distance between $\mathrm{f} 1$ and $\mathrm{f} 2$ that is $\mathrm{d}(\mathrm{f} 1, \mathrm{f} 2)$ should be greater than $\mathrm{d}(\mathrm{f} 1, \mathrm{r})$ and $\mathrm{d}(\mathrm{f} 2, \mathrm{r})$. 3. We must find the link stability.

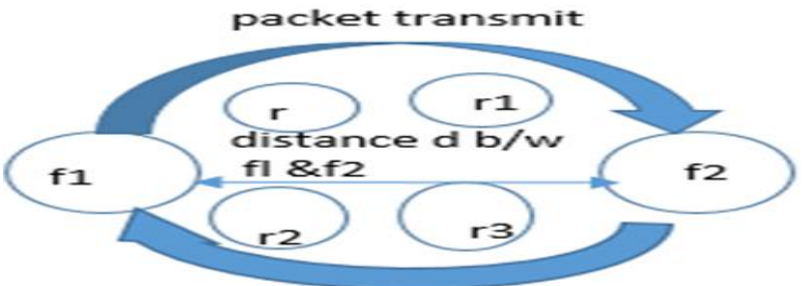

Fig5: Retransmission Region 


\section{Implementation Steps}

Multicast is a technique which is used to describe the communication where a piece of information is sent from one to many and many to many. In this case there are more than one sender, and the information is distributed to a group of receivers (theer may be no receivers, or any other number of receivers).

Multicast protocols select one or more than a path to reach the destination. They are better for load balancing than unicast protocols. Multicast routing which can improve the communication efficiency and they are reliable, reduce frequent routing update robust and as a result it reduces control overhead, improves data transmission rate, the network bandwidth is increased and the energy is saved. The list which can be forwarded is modified by finding frontier node same like unicast scheme.

\subsection{Multicast Proactive Routing Protocol (MPRP)}

- Every source node has complete knowledge of how to promote data packets to any node in the network at any time. In this network, tree based multicast protocol is used for forwarding the data packet from basis node to destination node.

- SNR calculation is done at MAC Layer. When received SNRj is less than $\mathrm{SNR}_{\min }$ threshold, the nodes that are decoded successfully while receiving help message is called super node.

\subsection{Forwarder List Update}

- The source node sends the route request packet it includes batch size, forwarder list size, packet number and forwarder number.

- Each intermediate nodes which are in the forwarded lidt, updates all these information.

- Each nodes calculate the channel gain between transmitter and super node, available bandwidth, Link Cost and also the multi hops for each path.

- Find the frontier node of the batch. Then the frontier has cached its first separation of packets in the present batch which are sent together from a given forwarder.

- When forward this subset of packet, the Outage probability is calculated then the routing module indicates there is a new method to the destination, it replaces the segment of the original forwarder list from itself to the receiver.

- Nodes which are sent forwarded list updates the link cost based on point to point connection, and send request packet along with available bandwidth information.

\subsection{Information Retransmission}

Nodes which are not found on the forwarder list but are placed between these two listed forwarders to retransmit data packets if the downstream forwarder has not received these packets successfully. If may be multiple such nodes connecting a given pair of listed forwarders, O_CORMAN coordinates retransmission among them and added these nodes into forwarder list successfully.

- All the forwarding nodes will calculate SNR value of its received packet, its Link cost(minimum transmission power) with available bandwidth, residual energy, amount of hops, are also included in the route request pkt.

- When the destination node receives the route ask for packet, it selects the best path based on these four parameters.

- Once a best path is selected, the destination node sends a route reply packet to the source node. Source forward the packets into destination. It rejects the old path and picks up the new path for data communication.

\section{Performance Analysis}

In this section, we discussed NS-2.32 simulation result of cooperative multicast O-Corman with unicast O-Corman in case of average consumed power, throughput, Control Overhead, and Packet Delivery Ratio. In the cooperative transmissions fails to deliver the message, we retransmit the message until the message is successfully delivered to the next hop. In our Mobile Ad hoc Network model nodes are spread in a random manner. Using opportunistic data transfer algorithm packets are forwarded from a source s and destination $\mathrm{d}$ nodes, with cooperative transmission

\subsection{Metrics}

We use the following metrics in evaluating the performance of the multicast routing protocols.average consumed power, throughput, Control Overhead, and Packet delivery ratio . 


\subsubsection{Packet delivery ratio}

It is computed as the ratio of entire number of unique packets received by the receivers to the entire number of packets transmitt by all sources times the number of receivers.

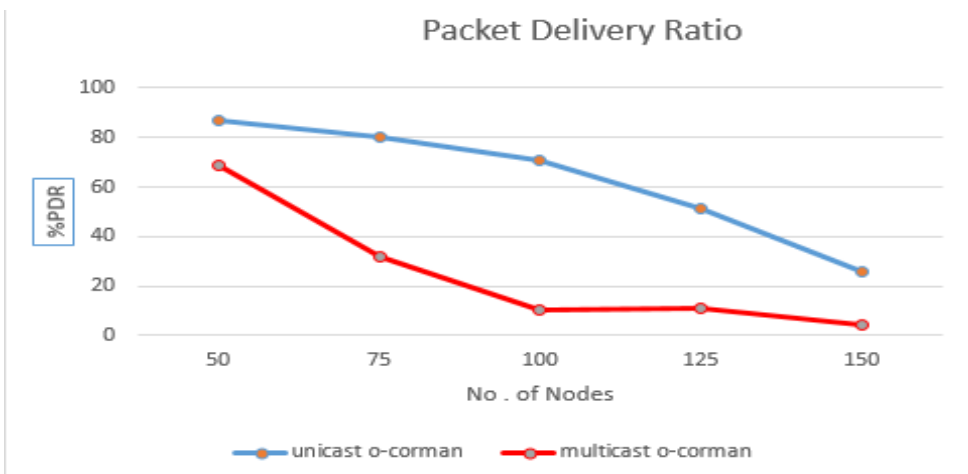

Fig 6: $\% \mathrm{PDR}$ of multicast and unicast o-corman

\subsubsection{Avg.Consumed Energy}

It is defined asthe energy consumption is averaged more than all nodes in the network.

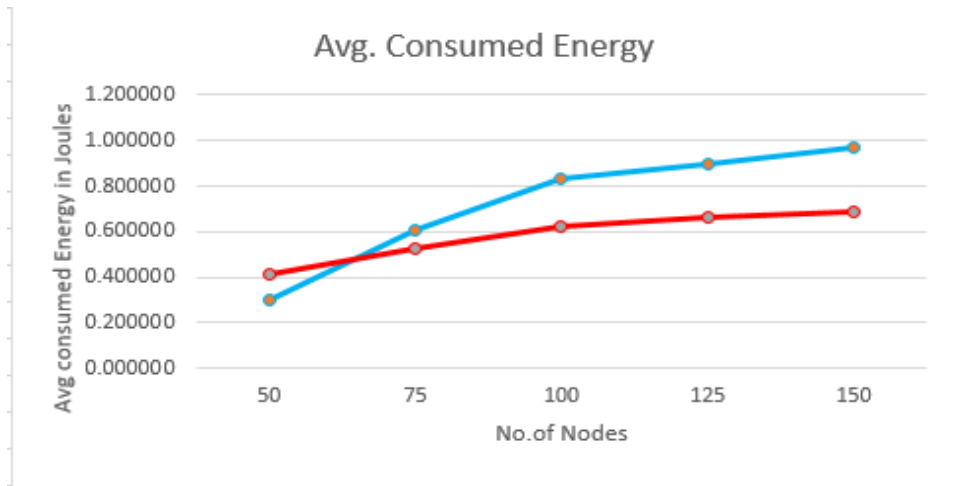

Fig 7: Avg. consumed energy of multicast and unicast o-corman

\subsubsection{Control overhead}

The control overhead is explained as the total number of routing control packets normalized by the total number of received data packets

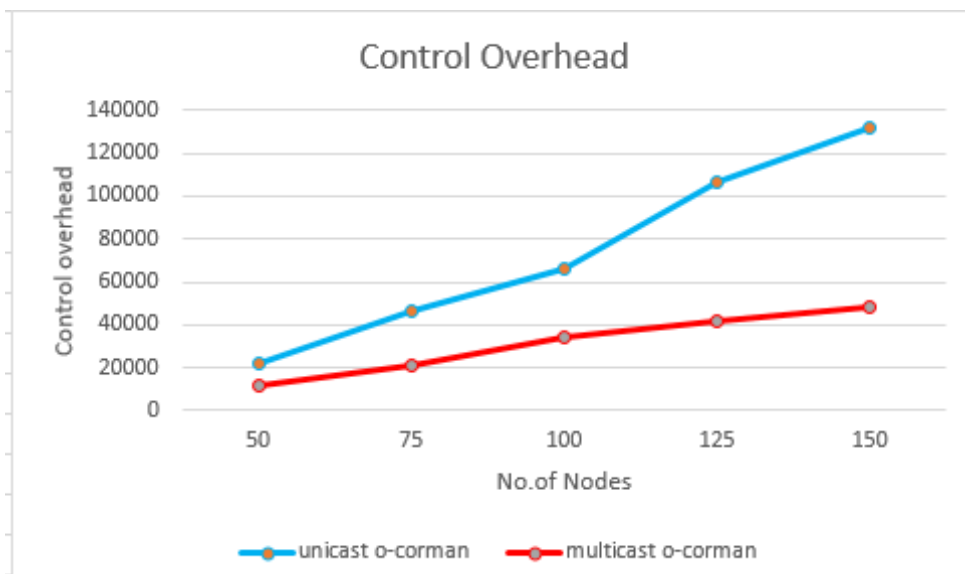

Fig 8: Control overhead of multicast and unicast o-corman

\subsubsection{Throughput}

It is explained as the total number of packets delivered over the total simulation time. The throughput increases when the connectivity is better. 


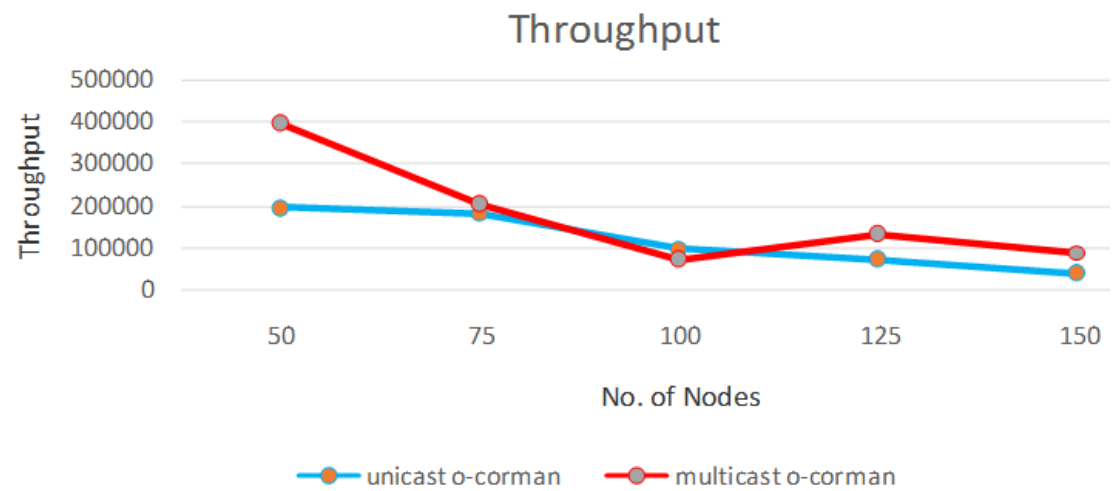

Fig 9: Throughput of multicast and unicast o-corman

\subsubsection{Average end-to-end delay}

The average end-to-end delay of data packets is the distance between the data packet generation time and the time when the last bit arrives at the destination.

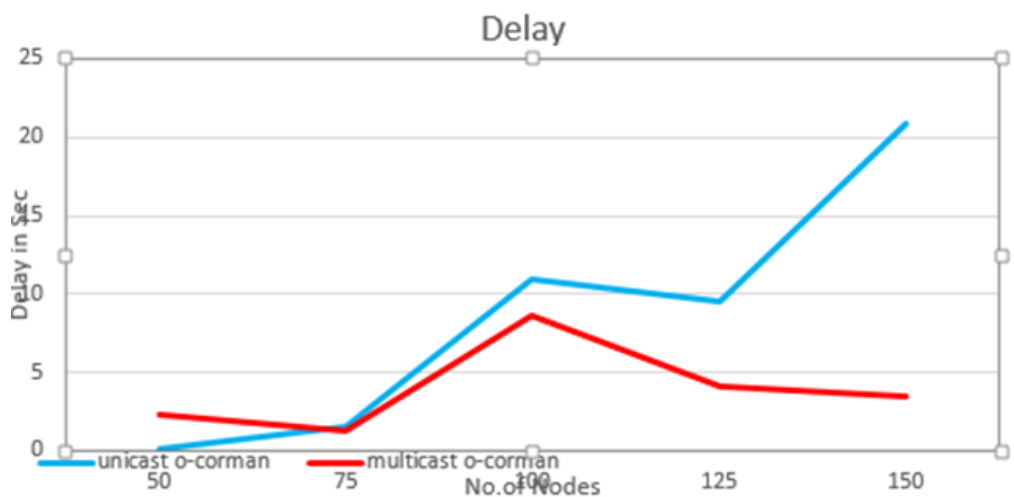

Fig10: Average end to end delay of multicast and unicast o-corman

The experiment is carried out by modifying a) Packet Delivery Ratio b) Throughput c) Delay d) Avg. consumed enery e) Control Overhead. The Proposed technique performs better than multi cast O- CORMAN in terms of, Throughput, Delay, Avg. consumed energy, and Control Overhead, but PDR is decreases in proposed scheme

\section{Conclusion And Future Work}

In this research paper, the proposed multipath routing scheme for mobile ad hoc networks, consist of three components. 1) MPSR - MULTIPATH PROACTIVE SOURCE ROUTING PROTOCOL, 2) large-scale live update of forwarder list, and 3) small-scale retransmission of missing packets. All of these explicitly utilize the distribution nature of wireless channels and are achieved via efficient cooperation among participating nodes in the network. It is also shown that the multipath routing for MANET have superior performance measured in delay, Avg. consumed energy, Throughput and Control Overhead. Similar to unicast the number of receiver is one, which has high PDR,but in our multicast scheme, when the number of receiver is more, more redundancy route exit, and PDR is high, this can be regard as in the future work. Also in future work has been done by using other multicast routing protocol that motive is to provide QOS guarantees, security.

\section{Acknowledgment}

This research paper is published with support of Anveshana educational and research foundation. I would like to thank sri Dr.S.Chakradhara goud for his comments that greatly improve the manuscript.

\section{References}

[1]. Rajaraman, R. - Topology Control and Routing in Ad hoc Networks:A Survey, SIGA News, vol. 33, pp. 60-73, June 2002.

[2]. Analysis of Different Routing Techniques for Opportunistic Data Transfer,\| International Journal of Computer Applications (0975 8887), volume 62 - No.5, January 2013 - Sheela Rani Arasu, and Immanuel JohnrajaJebadurai,

[3]. Path diminution in node - disconnected multipath routing for mobile ad hoc networks is unavoidable with single route discoveryll, International Journal Ad Hoc and Ubiquitous Computing - Ash Mohammad Abbas, and BijendraNath Jain,

[4]. Online]http://www.inetdaemon.com/tutorials/internet/ip/routing/single_vs_multi.shtm

[5]. Radio Disjoint Multi-PathRouting in MANET\|, in CEWIT 2005 Conference, 2005, pp 1-2 KoojanaKuladinithi, Carmelita Gorg and Samir Das 
[6]. The Design and Performance Evaluation of a Proactive Multipath Routing Protocol for Mobile Ad Hoc Networksll, at Heriot - Watt University in the School of Mathematical and Computer sciences, May 2012 - Ali AbdallaEtorban

[7]. ExOR: Opportunistic Multi-Hop Routing for Wireless Networks, „l in Proc. ACM Conference of the special Interest Group on Data Communication, Philadelphia, PA, USA, August 2005, pp. 133-14 -Ding, W., Biswas, S., and Morris, R..

[8]. SOAR: Simple Opportunistic Adaptive Routing Protocol for Wireless MeshNetworks,IEEE,vol. 8,2008,pp. 1622-1635 — R.Eric

[9]. "ExOR: opportunistic multi-hop routing for wireless networks," In Proc. of ACM SIGCOMM, Aug. 2005, - S. Biswas and R. Morris

[10]. Trading Structure for Randomness in Wireless Opportunistic Routing, , in Proc. ACM Conference of the Special Interest Group on Data Communication (SIGCOMM), Kyoto, Japan, August 2007, pp. 169-180 — S. Chachulski, M. Jennings, S. Katti, and D. Katabi

[11]. CORMAN: A Novel Cooperative Opportunistic Routing Scheme in Mobile ad Hoc Networksl, IEEE Journal on selected areas in Communications, Vol. 30, No. 2, February 2012 - Zehua Wang, Yuanzhu Chen and Cheng Li.

[12]. “ On-Demand multicast routing protocol in multihop wireless mobile networks," in ACM Mobile Networks and Applications, special issue on Multipoint Communication in Wireless Mobile Networks, 2000 - S.Lee, W.Su. and M. Gerla

[13]. E.Royer and C.Perkins, "Multicast operation of the ad-hoc-on-demand distance vector routing protocol,"in Mobile Computing and Networking, 1999, pp.207-218.

[14]. M. K. Marina and S. R. Das, "On-Demand MultiPath Distance Vector Routing in Ad hoc Networks", Proceedings of the Ninth International Conference on Network Protocols (ICNP\}, IEEE Computer Society Press, 2001, pp. 14-23.

[15]. J.Park, S.Moht and I.Chung \| Multipath AODV Routing Protocol in Mobile Ad Hoc Networks with SINR-Based Route Selection\|, International Symposium on Wireless Communication Systems, IEEE, 2008, pp:682-688.

[16]. L.Wang, L.Zhang, Y.Shu and M.Dong,„ Multipath source routing in wireless ad hoc networksl, Proceedings of Canadian Conference on Electrical and Computer Engineering, 2000, Vol 1, pp. $479-483$.

[17]. S. J. Lee and M. Gerla, "Split Multipath Routing with Maximally Disjoint Paths in Ad hoc Networksl, Proceedings of the IEEE International Conference on Communications (ICC), 2001, Vol 10, pp. 3201-3205.

[18]. X.Li, Ph.D thesis on "Multipath Routing and QoS Provisioning in Mobile Ad hoc Networks", Queen Mary University of London, 2006. 